\title{
Démocratie et évaluation des politiques publiques
}

\section{Jérôme Couture et Steve Jacob}

Université Laval

La théorie démocratique énonce que les élus élaborent et mettent en œuvre les politiques publiques souhaitées par les électeurs. En contrepartie, les électeurs " récompensent » ou « sanctionnent » les représentants sur la base de l'adéquation entre la performance publique et les attentes citoyennes (Couture et Jacob 2019). Lévaluation joue un rôle clé dans cette relation en tant quétape d'un processus où la légitimité des décideurs est questionnée. La notion de reddition de comptes est au cœur de la nouvelle gouvernance publique. Elle est le "Über-concept » ou le «buzz word» de ce début de 21e siècle (Flinders 2014). La reddition de comptes implique le contrôle de l'action gouvernementale par la divulgation d'informations sur la conduite et les choix des acteurs. Ici, le qualificatif " public " prend une importance particulière. La reddition de comptes doit se faire ouvertement, en toute transparence, plutôt que discrètement, derrière des portes closes (Bovens et al. 2014). Elle est rendue effective autant par des organismes spécialisés que par l'opinion publique. Dans ce contexte, la mesure de la performance est perçue comme un outil pouvant contribuer au renforcement de la confiance, de l'imputabilité et de la légitimité de la sphère politique et de la fonction publique face aux citoyens (Rouillard et Burlone 2011). Ainsi, l'analyse des facteurs qui influencent l'élaboration, l'appropriation et l'utilisation d'instruments de gestion de l'action publique par les institutions et les acteurs impliqués est un élément clé à étudier.

\section{L'ÉVALUATION AU CGER DE LA GOUVERNANCE DÉMOCRATIQUE}

Le lien entre lévaluation et la démocratie est ancien et plusieurs chercheurs se sont penchés sur la relation entre lévaluation et la démocratie. Plus précisément, ils étudient la manière dont lévaluation contribue (ou non) au bon fonctionnement de la démocratie. Dans cette optique, lévaluation constitue en elle-même une politique publique à évaluer, puisque le recours (ou le non-recours) à lévaluation par les organisations publiques varie fortement d'une juridiction à l'autre. Cette distinction sobserve au niveau du développement des capacités évaluatives (Bourgeois 2016), de la culture et de l'institutionnalisation de lévaluation (Jacob, Speer et Furubo 2015). À ce sujet, Beywl (2006) identifie quelques facteurs qui permettent de caractériser l'intentionnalité et la cohérence qui sous-tendent la pratique de lévaluation au sein de l'administration publique. Il s'agit du contexte juridique ou constitutionnel qui peuvent influencer la pratique, du niveau de réceptivité des gouvernements, de l'administration publique et des partis politiques, 
des routines administratives favorisant le recours aux données probantes, du nombre d'unité administratives dans le système gouvernemental qui procède à un cycle d'évaluation, de l'accessibilité générale de l'information au sein du système politique, de l'offre de formation en évaluation et de sa reconnaissance comme profession ainsi que du degré de diffusion des résultats des évaluations.

La gouvernance démocratique peut s'appréhender comme la résultante d'une compétition pour la représentation de divers intérêts ou valeurs au sein de la société. La représentation des valeurs portée à travers les programmes et les politiques publiques devient donc un élément fondamental à analyser pour saisir le lien entre l'évaluation et la démocratie. En effet, l'interprétation des informations et des résultats recueillis lors d'une évaluation peut devenir un moment critique du processus d'évaluation puisque la manière dont les valeurs sociétales sont prises en compte aura nécessairement un rôle à jouer à létape de l'interprétation. À ce sujet, deux pôles sopposent à propos de la place et du rôle des valeurs au sein des évaluations.

La typologie de Daniel Stufflebeam (2001) permet de distinguer les différentes traditions en évaluation du point de vue du rôle des valeurs et qu'on peut relier à certaines théories démocratiques existantes. Une première tradition qui se concentre sur l'efficacité et l'efficience des programmes insiste sur la distanciation avec les valeurs (values distanced). Cette tradition, souvent relier aux évaluations de troisième génération, correspond assez bien au modèle de la démocratie minimaliste de Joseph Schumpeter (1942) où les électeurs ont pour rôle de récompenser ou sanctionner les élus sur la base de la performance de l'action publique. Lévaluation peut ainsi favoriser l'imputabilité soit en améliorant les programmes et les politiques publiques ou encore en assurant une rétroaction aux électeurs par le biais de l'information à propos de la performance de l'action publique. Une seconde tradition prend plutôt en considération la relativité des valeurs (values relativistic) par le bais d'un dialogue continu avec les parties prenantes et une utilisation pragmatique des résultats de lévaluation. Cette tradition est plus près du modèle démocratique développé à travers le systémisme de David Easton (1965) où la conversion des préférences en décisions publiques repose sur lappui des citoyens et des groupes envers le gouvernement tout au long d'un mandat démocratique. Les évaluations dites de quatrième génération où l'on cherche à consulter les acteurs d'un programme afin de répondre à leurs besoins est en adéquation avec cette vision d'une démocratie qui demeure bien vivante entre les élections. Une troisième tradition incarne plutôt la priorisation des valeurs (values prioritising) à travers une consultation et une participation plus marquée des parties prenantes lors du processus évaluatif par le biais notamment de la délibération démocratique. Les évaluations de cinquième génération découlent assez directement du modèle de la démocratie délibérative, que l’on peut notamment relier aux travaux de Jurgen Habermas (1996), qui suggère que les décisions d'une communauté politique doivent être prises par la délibération publique de tous ses membres. Cette version de la théorie démocratique est fondée sur l'idée d'une communication entre les citoyens dans un espace public. Une quatrième et dernière tradition 
met l'accent sur les valeurs à intégrer à l'évaluation (values positioned) par le biais de l'empowerment des compétences en évaluation et de l'auto-évaluation des organisations. Cette tradition présente un lien fort avec la démocratie participative tel que décrite par Archon Fung (2003) qui encourage l'intervention directe du public dans les décisions dans le but d'enrichir les compétences des citoyens.

Lorsque l'on s'intéresse à la relation entre l'évaluation et la gouvernance démocratique, il ne faut pas perdre de vue le principe central de la séparation des pouvoirs. Au sein de l'appareil d'État, le contrôle de la production de nouvelles connaissances sur les programmes et l'accès à ces nouvelles connaissances permettent de caractériser des idéaux-type de démarches évaluatives. La typologie de MacDonald (1976) suggère trois types d'évaluation s'appuyant sur des relations de pouvoir différentes. L'évaluation bureaucratique est celle où l'évaluation est destinée à une agence gouvernementale déjà habilitée à allouer des ressources et à déterminer les orientations politiques. Le rôle de l'évaluateur se compare à celui d'un consultant en gestion et son travail n'est ni indépendant ni susceptible d’être examiné par le public puisque les mandataires de l'évaluation conservent un contrôle sur le produit de ce travail. L'évaluation autocratique est celle où lévaluation a aussi pour mandataire les agences gouvernementales, mais cette fois l'évaluateur conserve une indépendance en tant que conseiller externe et conserve aussi la propriété des produits de lévaluation. Son travail est validé par la communauté scientifique et peut servir, s'il est valide, à défendre des orientations politiques. Lévaluation démocratique a pour prémisse principale le droit du public à l'information. Les méthodes et les résultats doivent être présentés de manière à être accessibles à un public non spécialisé. Tous les participants à l'évaluation doivent aussi obtenir un accès et un contrôle sur la publication des données et des résultats de l'évaluation.

De plus, la focale avec laquelle les interventions publiques sont appréhendées offre des perspectives différentes sur la nature des politiques et programmes publics et à fortiori sur leur évaluation. Les principaux registres théoriques distinguent les politiques publiques prises sous l'angle de la résolution d'un problème et les politiques publiques abordées à travers lopinion publique (Leca 1996). Les analyses portant sur la gouvernance démocratique ont bien souvent tendance à confondre le public avec lélectorat et d'accorder ainsi un poids prépondérant à lopinion publique. Toutefois, le public concerné par une politique publique ou un programme spécifique est habituellement plus réduit que l'électorat. Ce public s'assimile davantage aux parties prenantes d'une politique publique, c'est-à-dire les groupes cibles présumés responsables d'un problème dont la prise en charge est publique, les bénéficiaires de la solution mise en oeuvre pour résoudre ce problème et les personnes lésées ou qui profitent indirectement de l'intervention publique. Cette vision classique de l'analyse des politiques repose sur une vision de résolution rationnelle des problèmes. En règle générale, dans les modèles dominants de l'analyse des politiques publiques, lélectorat intervient surtout lors de la mise à l'ordre du jour et peu au moment de lélaboration, de la mise en oeuvre ou de l'évaluation des politiques publiques. En ce qui concerne l'évaluation, 
les approches participatives sont un moyen dencourager la contribution directe et active d'une grande variété d'acteurs tels que les décideurs, les gestionnaires de projets, les bénéficiaires directs et indirects des interventions publiques, des représentants de la société civile et des citoyens (Daigneault et Jacob 2009; Jacob 2009 ; Jacob et Ouvrard 2009). Pour les tenants de la nouvelle gestion publique (New Public Management), comme Osborne et Gaebler (1992), l'introduction de procédures de participation des parties prenantes et le recours aux enquêtes d'opinion permettent aux citoyens, définis comme des usagers ou encore des clients, de préciser leurs besoins et d'exprimer leur satisfaction à propos des services publics. L'administration publique est ainsi encouragée à revoir ses relations avec les citoyens selon une vision de service à la clientèle qui serait alors de nature à favoriser l'imputabilité de la fonction publique. Pour les tenants d'une conception plus critique du rôle de la participation, c'est plutôt le droit de parole des acteurs concernés par une politique qui a un effet sur la réactivité et l'imputabilité de l'administration publique. Selon cette vision des choses, l'administration publique devrait davantage souvrir aux citoyens en créant des espaces de participation qui permettront de mieux connaître les différentes interprétations possibles d'un problème public. Par exemple, DeLeon (1992) propose de remplacer la rationalité instrumentale par l'analyse participative des politiques publiques, avec pour objectif avoué de replacer la subjectivité et les valeurs des acteurs au centre de l'action publique, en combinant la vision des experts et celle des acteurs de la société.

\section{DES DÉFIS À SURMONTER}

Les chercheurs qui étudient les relations qui unissent lévaluation et la gouvernance démocratique font face à plusieurs défis. La nature polysémique du terme " démocratie » représente le premier défi et explique également la multiplication des théories ou approches évaluatives qui se revendiquent d'une perspective « démocratique » (Hanberger, 2001). Un deuxième défi provient du fait que tous les acteurs de la société, qu'ils soient impliqués ou non dans lévaluation, possèdent leur propre idée de ce qu'une démocratie idéale représente et de ce qu'ils attendent de celle-ci. Ainsi, Hanberger (2006) souligne que les résultats d'une évaluation peuvent être influencés par la vision que l'évaluateur a de la démocratie. Lorsque vient le temps dévaluer la démocratie, il existe plusieurs angles d’approches. Le premier se concentre sur les institutions démocratiques (existence, rôle, fonctionnement). Le deuxième analyse le résultat des décisions prises par les gouvernants. Enfin, le troisième se concentre sur les valeurs fondamentales de la démocratie (liberté, transparence, etc.).

Lévaluation contribue au bon déroulement de la démocratie de plusieurs façons. En général, lévaluation contribue à la recherche de l'efficacité, de la pertinence et de la cohérence des interventions publiques. En ce sens, lévaluation vérifie que les interventions ciblent les bons groupes, quelles répondent à leurs besoins et qu'elles soient efficaces et efficientes (Sager, 2017).

Lévaluation permet de mettre en lumière les qualités et les défauts du système politique et d'alimenter l'agenda médiatique (Sager, 2017). Cet exercice critique 
permet un examen des institutions démocratiques et révèle les besoins de changements potentiels pour améliorer létat de la démocratie. À ce sujet, Stame (2006) en s'inspirant de l'exemple de l'Union européenne souligne que l'évaluation est un moyen d'apprécier les paliers de la gouvernance multi-niveaux. Selon elle, la réalisation d'évaluations à chacun des paliers offre une occasion de comparer les niveaux de gouvernance entre eux et ultimement d'aboutir à des conclusions qui pourraient améliorer la compréhension des systèmes et ultimement l'état de la démocratie.

Lévaluation contribue également à renforcer l'efficacité de la gouvernance du système démocratique. Cette fonction aide les gouvernants à comprendre ce qui est susceptible de mieux fonctionner dans une société. Une plus grande ouverture à la transparence de la part des gouvernants pourrait inciter les citoyens à participer plus activement à la vie en société (Stame, 2006). En effet, dans une société moderne, où la confiance des citoyens envers le gouvernement est en chute libre, l'évaluation est un moyen pour les élus de rétablir le niveau de confiance et de tenter de restaurer leur légitimité (Hanberger, 2001). Les citoyens peuvent se référer à une évaluation ou sa présentation par un journaliste pour être informés de l'action du gouvernement et dans certains cas, nettement plus rares, d'orienter leurs votes au moment des élections (Pollitt, 2006).

Dans l'arène politique, l'évaluation remplit plusieurs fonctions. D'un point de vue général, elle permet aux législateurs de comprendre ce qui peut être fait pour améliorer les interventions de l'État et éventuellement d'apprendre de leurs erreurs (Hanberger, 2006). L'utilisation des résultats d'une évaluation ne sera cependant pas la même en fonction de la place qu'occupent les élus en Chambre. Les ministres peuvent s'appuyer sur les évaluations de performance pour analyser l'efficacité d'un programme et en mesurer l'efficacité à court ou long terme (Pollitt, 2006). Les élus de lopposition de leur côté peuvent critiquer les actions gouvernementales en s'appuyant sur un avis professionnel externe (Hanberger, 2006). Qu'ils soient dans le parti gouvernemental ou dans l'opposition, les élus vont bien souvent employer des raccourcis lorsqu'ils vont citer les résultats d'une évaluation comme arguments dans la joute politique (Pollitt, 2006).

Comme nous venons de le voir, l'évaluation concourt de plusieurs manières au fonctionnement de la démocratie. Toutefois, l'évaluation est confrontée à plusieurs limites ou difficultés qui peuvent affecter son impact sur la vie démocratique des sociétés contemporaines. La première limite, d’ordre méthodologique, réside dans le fait qu'il est difficile d'établir la relation entre l'évaluation et la démocratie et par conséquent d'entreprendre des recherches spécifiques dans le domaine (Sager, 2017).

Une deuxième difficulté prend racine dans l'organisation de nombreux systèmes démocratiques qui reposent sur une gouvernance à plusieurs niveaux. La multiplication des paliers de gouvernement et donc d'intervenants dans l'élaboration et la mise en œuvre de programmes publics diluent le sentiment de responsabilité. Ainsi, les conclusions et les recommandations formulées dans les évaluations ne sont pas nécessairement lues par les personnes directement concernées et il y a un risque que personne ne se sente tenu d'agir (Hanberger, 2006). De plus, le citoyen qui est rarement au fait de la complexité des arrangements 
institutionnels et des subtilités dans la répartition des compétences est bien souvent dépourvu quand vient le temps de comprendre pourquoi les problèmes concrets qu'il vit ne sont pas résolus par les gouvernants.

Un troisième défi porte sur la diffusion et l'appropriation des connaissances évaluatives auprès d'un large public. Il s'agit ici d'un enjeu de transparence qui est au cœur de l'exigence démocratique. Cependant, dans la plupart des cas, lévaluation apparaît comme un enjeu de pouvoir (Eliadis, Furubo et Jacob 2011) qui reste entre les mains d'une « clique élitiste » (House, 2006). En effet, il est assez rare que les ministres, les parlementaires ou les citoyens prennent connaissance intégralement de rapports dévaluation. La principale raison qui explique cette situation est la longueur et la complexité des rapports dévaluation qui découragent le lecteur ou rendent inaccessible le contenu des rapports (Pollitt, 2006). Comment les évaluateurs peuvent-ils rendre le résultat de leur travail accessible, pertinent et surtout compréhensible à tous? Il n'y a pas de réponse évidente à cette question même si plusieurs auteurs présentent lévaluation comme un instrument qui concourt au débat démocratique. Dans la plupart des cas, les destinataires des évaluations demeurent les gouvernants qui peuvent en faire une lecture et utilisation partielle voire partiale. A ce sujet, les parlementaires vont parfois réclamer la réalisation d'une évaluation pour faire pression sur le gouvernement et le mettre dans l'embarras. Dans d'autres cas, les parlementaires préfèrent lire lévaluation qui répond à leur besoin immédiat (1981) et rejoint leur opinion (Sager, 2017). Ce biais de convergence se comprend aisément dans un système partisan centré sur la joute parlementaire. Cependant, si les évaluations qui ne sont pas en adéquation avec l'agenda politique ne sont pas prises en considération, le fonctionnement de la démocratie en est affecté et les interventions publiques qui sont développées ne s'appuient pas sur les données probantes. Des pistes de solutions ont été suggérées pour dépasser ce défi. Afin d'accroître l'attention qui est portée aux rapports dévaluation, il est important de tenir compte du timing, sans sacrifier les exigences de rigueur méthodologique, les évaluateurs devraient chercher à identifier des moyens leur permettant de fournir plus rapidement une information de qualité aux décideurs pour que celle-ci soit disponible au moment opportun dans le processus décisionnel (Pollitt, 2006).

\section{PRÉSENTATION DU SEGMENT THÉMATIQUE}

Ce segment thématique illustre les concepts de reddition de compte, de légitimité, de transparence, de participation et présente leurs répercussions sur lévaluation. Il met également laccent sur les dimensions de la communication politique qui sont moins souvent discutées dans la littérature en évaluation de programme, les évaluateurs se concentrant davantage sur les dimensions administratives ou de gestion des processus évaluatifs. En portant une attention particulière aux enjeux politiques de lévaluation, les deux contributions rassemblées dans ce segment identifient des enjeux novateurs pour les praticiens et les chercheurs actifs dans le domaine de l'évaluation.

Catherine Fallon en s'appuyant sur le cas de municipalités en Wallonie (Belgique) questionne la relation entre la démocratisation et la recherche de l'efficience. 
Sous l'impulsion des réformes de la nouvelle gestion publique, les municipalités wallonnes se sont dotées de nouveaux mécanismes de gestion stratégique. Afin de pallier les dimensions technocratiques de ces dispositifs, quelques municipalités ont introduit des mécanismes participatifs. Cet article explore donc la relation qui existe entre les acteurs politiques, administratifs et les citoyens dans cette nouvelle configuration administrative.

Lisa Birch et Steve Jacob analysent la " résultologie " (Deliverology), une méthode de suivi des réalisations des promesses électorales, qui se développe dans plusieurs états depuis l'avènement de la nouvelle gouvernance politique. Cette pratique de gestion publique a été popularisée par Michael Barber (2016) dans son ouvrage How to run a government so that citizens benefit and taxpayers don't go crazy. En se concentrant sur la réalisation des engagements électoraux formulés par le parti gouvernemental lors de la campagne électorale, la « résultologie » s'inscrit dans le courant des approches de gestion axées sur les résultats. Cependant, comme lexpliquent les auteurs, les tenants de la résultologie recyclent des concepts qui sont utilisés depuis longtemps par les évaluateurs de programme. Cet article permet de mieux comprendre les similitudes et les différences qui existent entre lévaluation et la « résultologie ».

\section{REMERCIEMENTS}

Nous remercions le Fonds de recherche du Québec - Société et culture (FRQ-SC) pour son soutien financier ainsi qu'Anik Dupuis, auxiliaire de recherche au Centre d'analyse des politiques publiques (CAPP) pour sa contribution à la recherche documentaire.

\section{RÉFÉRENCES}

Barber, M. (2016). How to run a government so that citizens benefit and taxpayers don't go crazy. London: Penguin Books.

Beywl, W. (2006). The role of evaluation in democracy: Can it be strengthened by evaluation standards? A European perspective. Journal of MultiDisciplinary Evaluation, 6, $10-29$.

Bourgeois, I. (2016). Performance measurement as precursor to organizational evaluation capacity building. Evaluation Journal of Australasia, 16(1), 11-18.

Bovens, M., Goodin, R.E. et Schillemans T. (éds.). (2014). Public accountability. Dans The Oxford Handbook of Public Accountability (pp. 1-20). Oxford: Oxford University Press.

Couture, J. et Jacob S. (éds.). (2019). Démocratie et politiques publiques, Québec : Presses de l'Université Laval.

Daigneault, P.-M. et Jacob S. (2009). Toward accurate measurement of participation: Rethinking the conceptualization and operationalization of participatory evaluation. American Journal of Evaluation, 30(3), 330-348.

DeLeon, P. (1992). The democratization of the policy sciences. Public Administration Review, 52(2), 125-129. 
Easton, D. (1965). A framework for political analysis. Englewood Cliffs, NJ: Prentice-Hall.

Eliadis, P., Furubo, J.-E. et Jacob, S. (éds.). (2011). Evaluation: Seeking truth or power?, New Brunswick, NJ: Transaction Publishers.

Flinders, M. (2014). The future and relevance of accountability studies. Dans M. Bovens, R.E. Goodin et T. Schillemans (éds.), The Oxford Handbook of Public Accountability (p. 661). Oxford: Oxford University Press.

Fung, A. (2003). Survey article-Recipes for public spheres: Eight institutional design choices and their consequences. The Journal of Political Philosophy, 11(3), 338-367.

Habermas, J. (1996). Three normative model of democracy. Dans S. Benhabid (éds.), Democracy and Difference (pp. 21-20). Princeton: Princeton University Press.

Hanberger, A. (2001). Policy and program evaluation, civil society, and democracy. American Journal of Evaluation 22(2), 211-228.

Hanberger, A. (2006). Evaluation of and for democracy. Evaluation, 12(1), 17-37.

House, E.R. (2006). Democracy and evaluation. Evaluation, 12(1), 119-127.

Jacob, S. (2009). Opération chloroforme ou la réinvention de l'État rationnel. Lévaluation et les données probantes. Criminologie, 42(1), 201-223.

Jacob, S. et Ouvrard, L. (2009). Lévaluation participative. Avantages et difficultés d'une pratique innovante. Cahiers de la performance et de l'évaluation, no 1. Québec, Perfeval.

Jacob, S., Speer, S. et Furubo, J.-E. (2015). The institutionalization of evaluation matters: updating the international atlas of evaluation 10 years later. Evaluation. The International Journal of Theory, Research and Practice, 21(1), 6-31.

Leca, J. (1996). Ce que l'analyse des politiques publiques pourrait apprendre sur le gouvernement démocratique. Revue française de science politique, 46(1), 122-133.

MacDonald, B. (1976). Evaluation and the control of education. Dans D. Tawney (éds.), Curriculum Evaluation Today: Trends and Implications (p. 125-136.). London: MacMillan Education.

Pollitt, C. (2006). Performance information for democracy: The missing link?. Evaluation, $12(1), 38-55$.

Osborne, D. et Gaebler, T. (1992). Reinventing government. Reading, PA: Addison-Wesley.

Rouillard, C. et Burlone, N. (éds.). (2011). L'État et la société civile sous le joug de la gouvernance. Québec : Presses de l'Université Laval.

Sager, F. (2017). Policy Evaluation and Democracy: Do they fit?. Evaluation and Program Planning. 69, 125-129.

Schumpeter, J.A. (1942). Capitalism, socialism, and democracy. New York: Harper \& Brothers.

Stame, N. (2006). Governance, democracy and evaluation. Evaluation, 12(1), 7-16.

Stufflebeam, D. (2001). New directions for evaluation: Evaluation models. San Francisco: Jossey-Bass. 